\title{
A Case of Metachromatic Leukodystrophy with an Emphasis on Morphology
}

\author{
Gennady Bisaga ${ }^{1}$, Olga Gaykova ${ }^{1}$, Ljudmila Onishchenko ${ }^{1}$, Alexey Sobolev ${ }^{1}$, Tatiana Bukina ${ }^{2}$, Ekaterina \\ Zakharova $^{2}$, Alexey Sokolov ${ }^{3}$, Vera Ellinidi ${ }^{4}$, Alexey Popov $^{1}$, Igor Litvinenko ${ }^{1}$ \\ ${ }^{1}$ The Clinic of Nervous Diseases of S.M.Kirov Military Medical Academy, St. Petersburg, Russian Federation \\ ${ }^{2}$ FSBI Research Centre of Medical Genetics of the Russian Academy of Medical Sciences St. Petersburg 1, Russian \\ Federation \\ ${ }^{3}$ The Department of Extracorporal Detoxication of S.M.Kirov Military Medical Academy, St. Petersburg, Russian \\ Federation \\ ${ }^{4}$ The Laboratory of Clinical Immunology of the Nikiforov Russian Center of Emergency and Radiation Medicine of \\ the Ministry of Emergency Situations, St. Petersburg, Russian Federation \\ Email: bisaga@yandex.ru
}

\begin{abstract}
Clinical, imaging, laboratory and morphological data of a metachromatic leukodystrophy (MLD) patient were analyzed retrospectively. The clinical picture consisted of progressing pyramidal, cerebellar, brain stem, optical, mental, and bowel and bladder disturbances, and epileptic seizures. Large symmetric periventricular T2 lesions were seen on the magnetic resonance imaging brain scans. Unspecific lesions of 11C-methionine storage were found by brain positron emission tomography. Light microscopy of brain biopsy did not reveal any morphological changes specific for MLD, but some unusual pictures of myelinopathy in many myelin fibers were detected by electron microscopy. Biochemical analysis of lysosomal ferments or their activator proteins and deoxyribonucleic acid (DNA) diagnostics were conclusive for the diagnosis of MLD. Differential diagnosis was performed to identify various leukodystrophy forms and other central nervous system diseases.
\end{abstract}

Keywords: Metachromatic leukodystrophy, brain biopsy, light microscopy, immunohistochemistry, electron microscopy, DNA-diagnostics.

\section{Introduction}

Leukodystrophies (Greek: leucos "white" + dystrophy) are a group of hereditary diseases with progressing dystrophy of the brain and spinal cord white matter. Metachromatic leukodystrophy (MLD) is a biochemical and/or molecular-genetic defect $[2 ; 14]$. The genetic defect leads to disturbance of formation and/or fast breakdown of myelin. The previous morphological brain research had revealed neurodegeneration and spongiose degeneration of the leukodystrophy white matter [14] with symmetrical extensive confluent demyelination lesions seen on magnetic resonance imaging (MRI) and active microglia proliferation with a phagocytosis of the myelin destruction products [2]. Some cases showed impairment of the spinal cord and peripheral nerves $[1 ; 3]$.

In our article an MLD patient aged 25 was surveyed and discussed. The study included serum and cerebrospinal fluid (CSF) examination, brain MRI and positron emission tomography (PET), light microscopical, immunohistochemical and electron microscopical brain periventricular lesions biopsy investigation, and genetic typing.

\section{The Case Description}

The patient C., 25 y.o., was complaining of considerable decrease in visual acuity, "oscillatory movements" of eyeballs when looking all over the sides, weakness and spasticity in lower extremities, loss of memory and attention, periodical urine incontinence. The first symptoms appeared at the age of 22 and they were weakness in legs and decreased visual acuity. At the age of 23 , the visual impairments 
progressed, walking unsteadiness became stronger, urine incontinence appeared. These symptoms were the same during the following two years.

From the history it was known that in the infancy the patient had had a delay of the motor development and started walking at the age of 1.5-2 years. At the age of 9 months occasional "oscillatory movements" of eyeballs, and the epileptic seizures with tonoclonic spasms had been diagnosed. The patient had been observed domiciliary as having infantile cerebral paralysis and consequences of the birth brain trauma with the left-side hemiparesis. At the age of 24 the patient had been treated with plasmapheresis (3 procedures a week) and methylprednisolone administered intravenously in a cumulative dose of $1500 \mathrm{mg}$ in 3 days, which resulted in an insignificant short-term improvement of the vision.

During the last hospitalization at the age of 25 no somatic disorders were found. The following symptoms of cerebral white matter, brain stem and cranial nerves disturbances were registered: a strong vertical and horizontal congenital nystagmus, a change of visual acuity to 0.2 on the right and to 0.3 on the left, staccato speech, lip reflex, moderate lower pyramidal paraparesis, Babinsky sign on the right, spastic-paretic and atactic gait. Strong kinetic tremor and dysmetria predominantly on the left side were registered on coordination testing. The sensation was normal. Cognitive functions were disturbed moderately, which were manifested in the decreased interest in surroundings and limited vocabulary.

Differential diagnosis was needed due to unexplainably fast increase of the disease symptoms at the age of 22 (especially, pyramidal, cerebellar, stem and pelvic), that could mimic demyelinating diseases, particularly primary progressing multiple sclerosis (MS), and others (systemic rheumatic diseases, vasculitidies, herpetic or bacterial infections and chronic disseminated encephalomyelitis).

\subsection{Laboratory Results}

Free light chains of immunoglobulins in the CSF slightly exceeded the norm: kappa-chains were 0.061 $\mu \mathrm{g} / \mathrm{ml}$, lambda-chains were $0.0006 \mu \mathrm{g} / \mathrm{ml}$ ( $\mathrm{N} 0-0.04$ and 0-0.02 $\mu \mathrm{g} / \mathrm{ml}$, respectively).

The serum analyses showed a little excess of lymphocytes (39.0; N 19.0-37.0), a decrease of T-killers (the absolute quantity was 343; N 450-850, the relative one was 22; N 23-40) and an elevated relative number of NK-cells (21; N 9-19). The research of serum cytokines detected only one disturbance - a decrease of the induced production of IL-6 (142 pg/ml; N 1000-1300). The level of IgM antibodies to myelin basic protein was slightly increased: 1.30 (N 0.00-1.10). Serum nervous growth factor (BDNF) was decreased $(5.5 \mathrm{ng} / \mathrm{ml} ; \mathrm{N} 15-30)$. Among the common serum immunoglobulins only IgA was slightly elevated: $5.06 \mathrm{~g} / \mathrm{l}$ (N 0.90-4.50). Superoxide dismutase and catalase of red cells were decreased (151.0 units/l; N 164-240 and 4.5 units/1; N 5.2-10.5, respectively).

Serum lactate (4.30 mcM/l; N 1.33-1.80) and serum ammonia (167 mcM/l; N 10-47) levels were sharply elevated that could be explained by hypoxia, similar to that in case of mitochondrial disease, in which the malfunction of energy metabolism is generally accepted.

Thus, the completed tests did not indicate any signs of either autoimmune diseases, particularly MS, acute or chronic infectious or hereditary diseases.

\subsection{Instrumental Testing Results}

Ophthalmological examination revealed bilateral atrophy of optic nerves, concentric narrowing of visual field, congenital nystagmus and astigmatism. The data showed a degenerative process in the optic nerves; central nervous system CNS inflammatory demyelinating process could not be excluded.

Brain MRI: large symmetric periventricular hyperintensive signals were visualized on the T2-weighted images. Radiologists considered them the signs of possible demyelinating process (Fig.1), but contrast images were not made. 


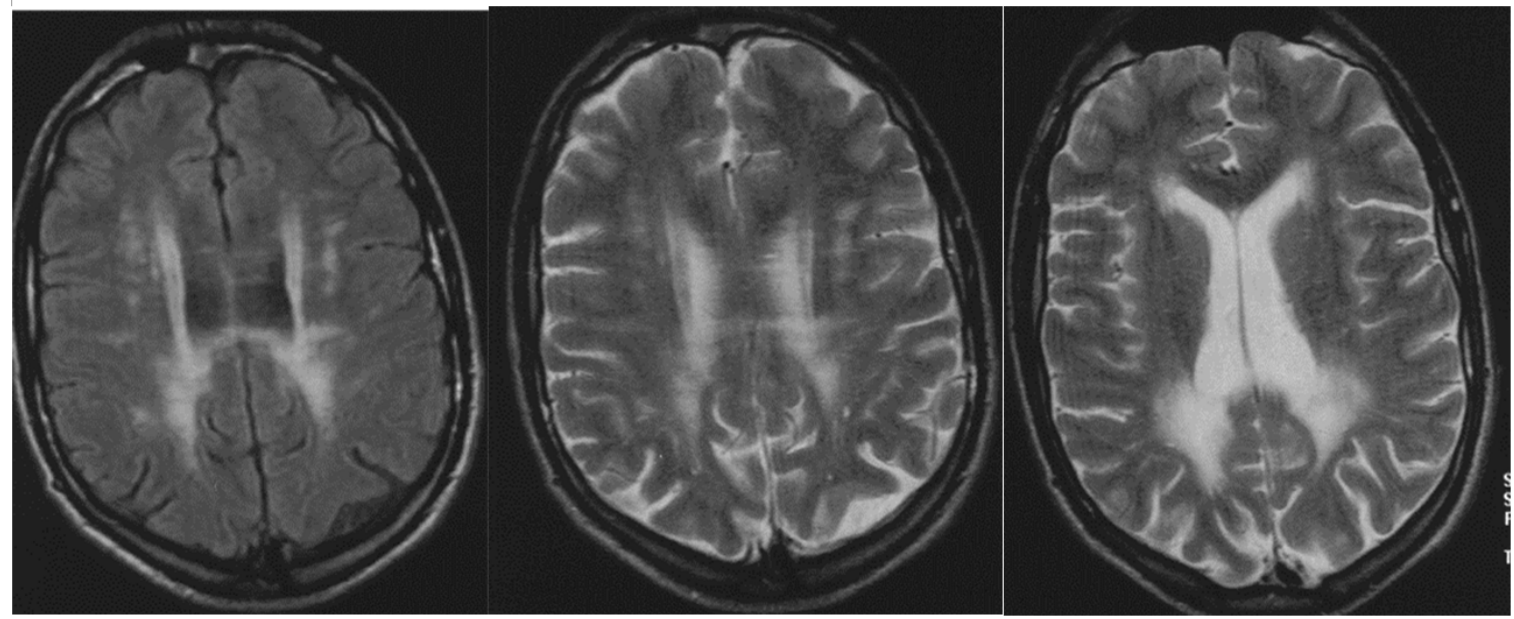

Figure 1. Brain MRI, T2-waighted images. Symmetrical multiple T2 lesions in the periventricular and deep white matter.

11C-methionine (11CM) PET detected a lesion with increased accumulation of $11 \mathrm{CM}(\mathrm{IN}=1,4)$ in the middle of the left central gyrus and in the subcortical area $21^{*} 14^{*} 15 \mathrm{~mm}$ (Fig.2). However the accumulation of $11 \mathrm{CM}$ in the zone of interest on the early vascular phase was not detected. Thus, PETexamination did not completely exclude tumorous genesis of 11CM accumulated lesion of the brain, and that made us carry out the stereotactic biopsy of the lesion.
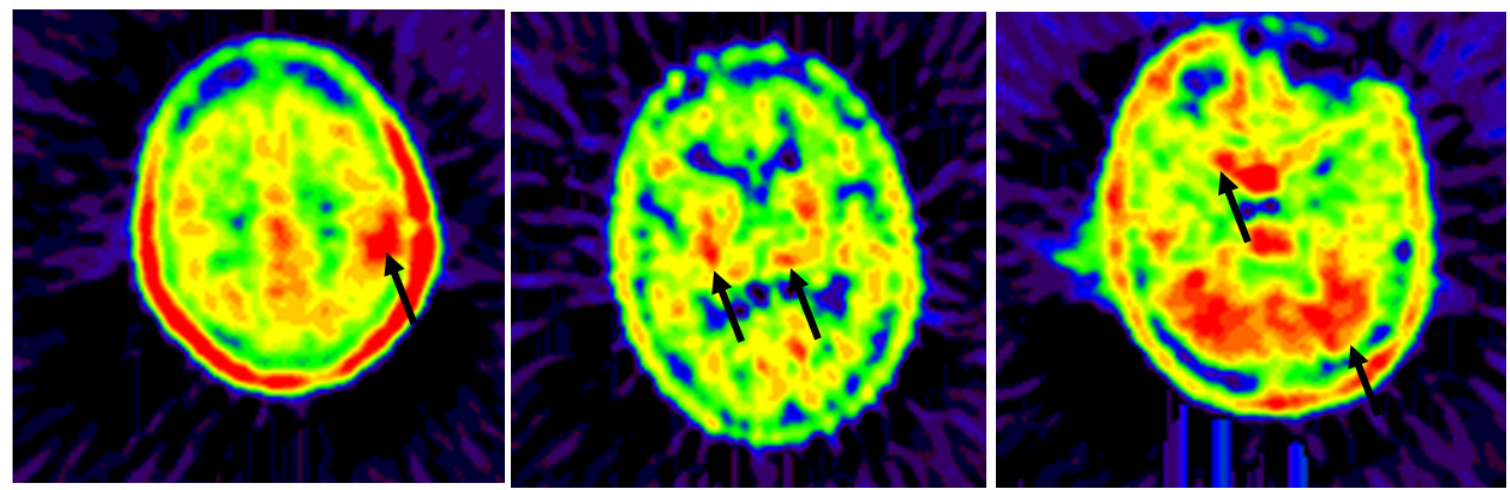

Figure 2. 11C-methionine PET of the brain. An irregular shape focuses of the radiopharmaceutical accumulation in the subcortical zones, in the central left gyrus and cerebellum (arrows).

After a surgical manipulation, the brain biopsy material was prepared for the light microscopy using standard methods [Merkulov G.A., 1969]. Histological paraffin sections were stained by hematoxylin and eosin by the Van-Gieson, Spielmeyer, Nissl and Mallory methods and PAS reaction.

The biopsy material was prepared for electron microscopy by standard methods [16]. Semithin eponaraldit sections were stained by toluidine blue by the Nissl method and were studied using a light microscope. The ultrathin sections were analyzed using an electron microscope LEO-910 (Germany). Derived electron slices were scanned for further detailed analysis.

\section{Brain biopsy results}

Light microscopy of the brain white matter detected dystrophic and degenerative changes without any signs of inflammation, demyelination or atypical growth. Hematoxylin and eosin staining revealed that neuropil was irregularly thinned out right up to small cavities of a rough-edged uneven form (Fig.3A, B). The quantity of the glial cells was insignificantly increased, but the majority of astrocytes were "fat" with large cytoplasmic eosinophilic granulosity and eccentrically located nucleus. Nucleus chromatin condensation in some oligodendrocytes reminded of the picture of apoptosis initiation. 


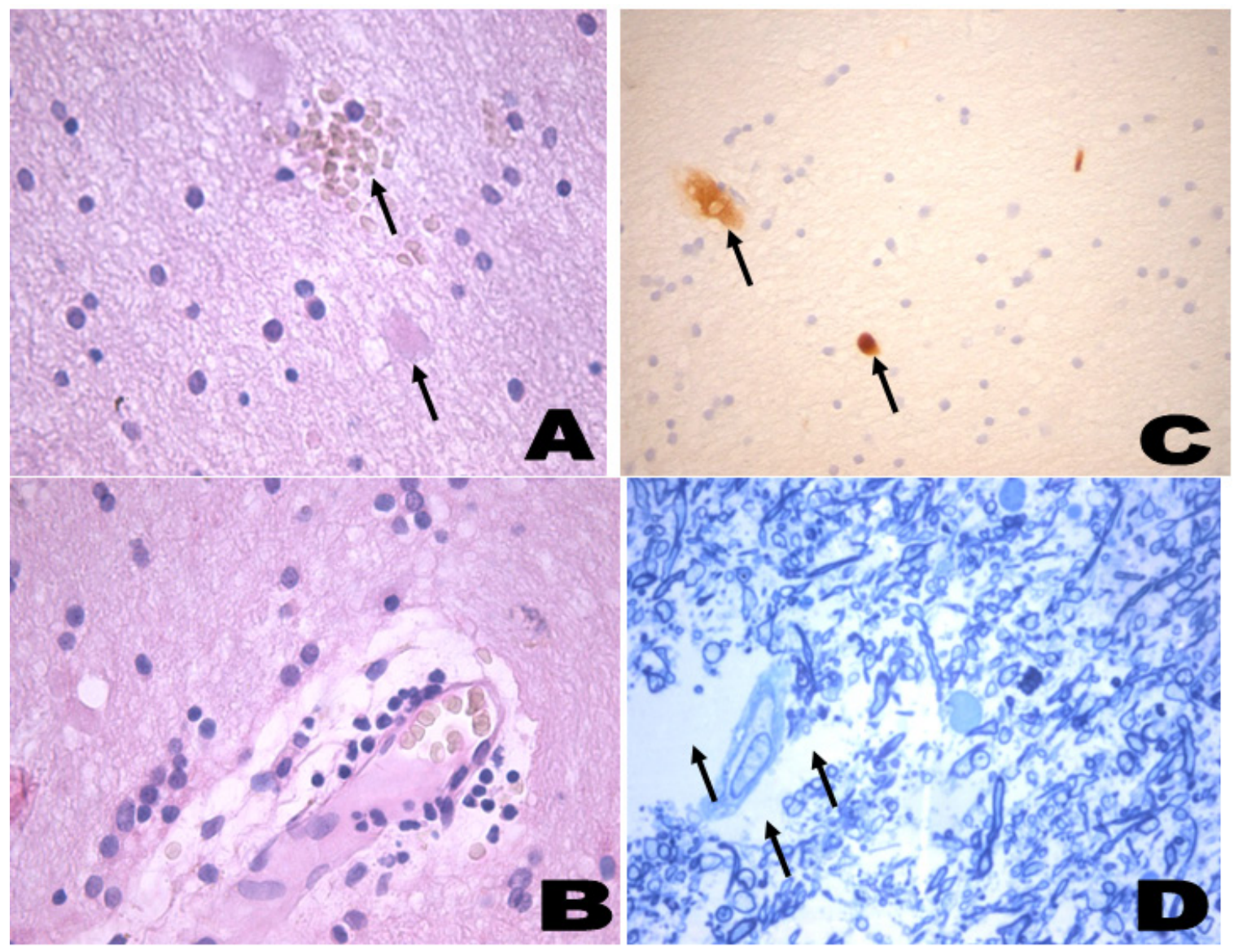

Figure 3. Light microscopic examination of the brain biopsy lesion in the periventricular white matter. A: Small haemorrhages, amorphous and moderate eosinophilization without clear borders with the diameter of 50-100 $\mu \mathrm{m}$ (arrows); B: Vessels with swollen endothelium; the lymphocytes and macrophages are located perivascularely; C: Amyloid inclusions and sparse neuropile (arrows). D: Vessels with swollen endothelium and lysis of the pericapillary white matter (arrows).

A,B: Hematoxylin and eosin stain - x630; C: Congo-Rot stain - x400; D: Semi-thin section stained with the toluidine blue by the Nissl method $-\mathrm{x} 400$.

The vessel endothelium was slightly swollen, and around the separated arteries there was detected an insignificant lymphomacrophagal infiltration (Fig. 3B). Also, there were amorphous moderately eosinophilic lumps without distinguished boundaries of $50-100 \mathrm{mcm}$ in diameter similar to amyloid plaques in case of dementia (Fig. 3C). Vessels with swollen endothelium and lysis of the pericapillary white matter (arrows) were found (Fig. 3D).

Electron microscopic examination revealed atypical structure of karyo- and cytoplasm in the sporadic extant oligodendrocytes. Their weakly or moderately osmiophilic karyoplasm had unusually homogeneous chromatin structure and the cytoplasm was either absolutely transparent or with some perinuclear organoids that were found on one of the cellular poles. Also, there were cells with the cytoplasm containing enough typical or modified organoids, such as vacuolated mitochondria (along with mitochondria having normal strong matrix and cristae) and vacuolated tubules of the granular endoplasmic reticulum. In the studied biopsy material both axono- and myelinopathy occurred (Fig. 4A, B). 

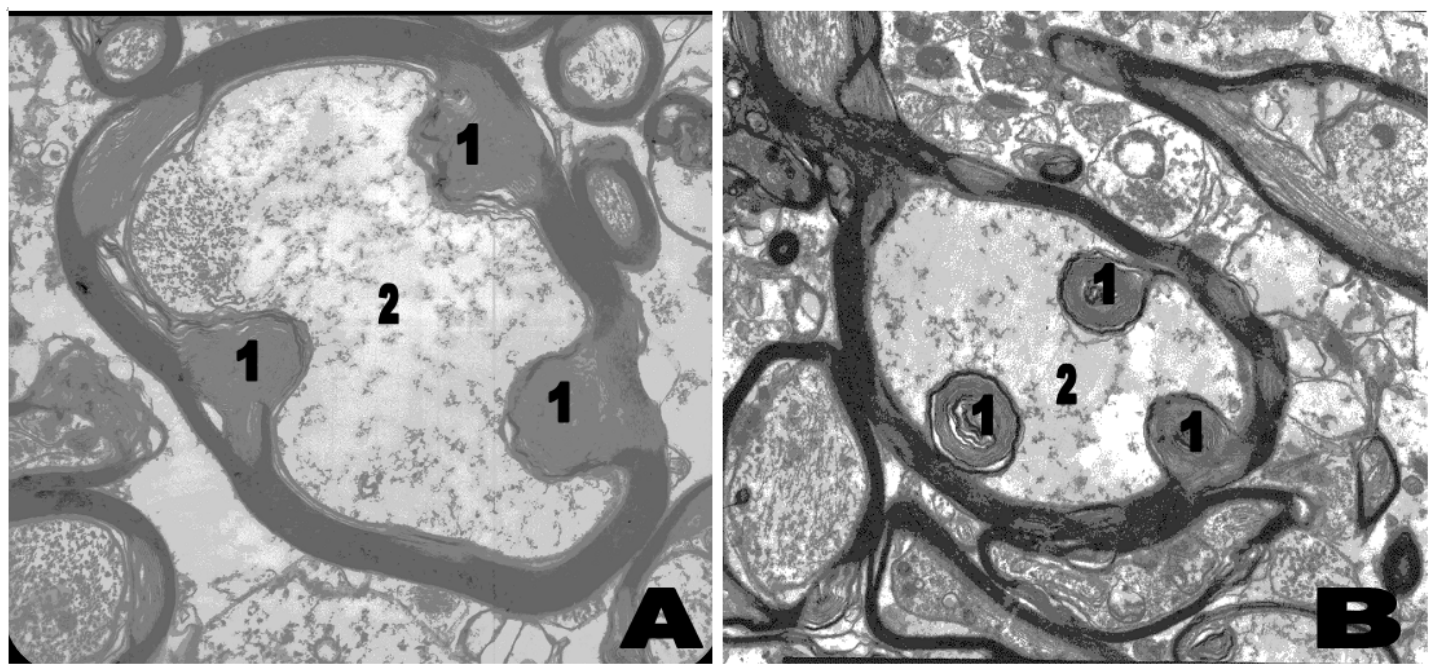

Figure 4. Electron microscopic examination of the brain biopsy lesion in periventricular white matter with myelino- and axonopathy of the myelin fibres.

A. Large myelin fiber with forming periaxonal outgrowths of the broken myelin lamellae (1) is a distinctive manifestation of myelinopathy. There is a moderate axonopathy of the light type (arrows) x7 000

B. Generated periaxonal outgrowths of myelin (1) are the manifestations of myelinopathy. There is also a moderate axonopathy of the light type (arrows) x5 000

Axonopathy (of the light type) is found very rarely in myelin fibers and more often in nonmyelinated fibers. The main distinctive criterion for myelin modification (rearrangement) was the outgrowth from broken lamellae both outside and more often inside of myelin sheath. In the latter case, the axon was compressed or completely replaced by such growth (Fig.4A, B). Myelin fragments were detected in the cytoplasm of various oligodendrocytes that indicated myelin phagocytosis of those cells. There were also macrophages containing a lot of strong osmiophilic granules and broken myelin fibers in their cytoplasm (Fig. 5A). Moreover, we detected sharp structural failures of synaptic contacts that indicate synaptic transmission disturbance (Fig.5B).
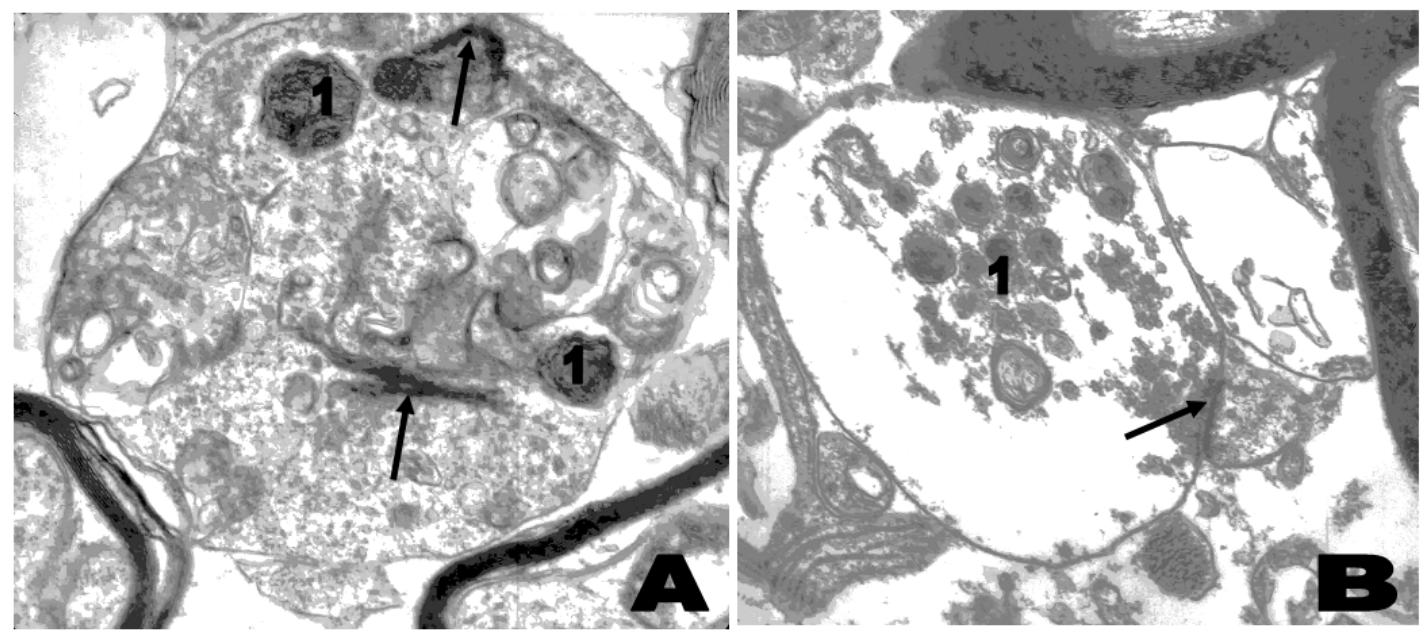

Figure 5. Electron microscopic examination of the brain biopsy lesion in periventricular white matter.

A. An active macrophage with large quantity of phagosomes (1), lysosomes and broken myelin fibers in cytoplasm (arrows) x6300

B. Axo-dendritic destructively modified synapse with a packed synaptic gap (arrow), disturbed synaptic vesicles on both sides of the gap (arrows) and strongly modified mitochondriae and organoids in the dendrite (1) x16 000 
In addition to the mentioned above, the solitary clusters of erythrocytes were found far away from vessels, and it was regarded as indirect indication of the blood brain barrier disturbance. At the same time, there were no clear disturbances of the vessels basal membranes. However, some capillaries had apparent luminal narrowing and others had stasis of erythrocytes.

Specific biochemical and molecular-genetic diagnostics results

The most probable concurrent diseases were excluded by biochemical and genetic methods: 1) Xlinked adrenoleukodystrophy: the level of very long chain fatty acids was normal, 2) leukoencephalopathy with cerebellum and stem damage, and high lactate level: the analysis of the gene DARS2 did not detect any pathogenic mutation.

The measurement of enzyme activity of arylsulfataze A (ASA) was carried out by the means of potassic paranitrocatecholsulphate salt [Sommerlade H.J. et al., 1994]. There was detected a significant decrease of the ASA activity in the patient's blood leukocytes. The ASA activity was $28 \mathrm{nM} / \mathrm{mg} / \mathrm{h}$, that is about $10 \%$ of norm $(\mathrm{N} 210.2+/-83.7 \mathrm{nM} / \mathrm{mg} / \mathrm{h})$, which strongly confirmed the diagnosis of MLD. The analysis of frequent mutations of the ASA gene detected a replacement of p.536T $>\mathrm{G}$ which in its turn led to a replacement of isoleucine by serine in the 179th protein chain position (p.Ile179Ser) in heterozygous state. A search of the second mutation was not carried out.

\section{Discussion}

MLD is an autosomal-recessive myelin exchanges disease, characterized by the accumulation of galactosyl-sulphatide (cerebroside-sulphatide) in the central and peripheral nervous system [4]. In some variants of MLD the disturbance of the gene and its encoded enzyme ASA that detached sulphatic group from sulphatide were found [22; 13].

In a typical clinical case of MLD usual symptoms are mental (depression, schizophrenia-like psychosis, chronic alcoholism). Commonly, the disease starts after puberty and slowly progresses. Even so, the disease symptoms grew rather quickly in our patient. He lost the ability to take care of himself, vision and mental capacity and eventually, as a result of complete disability, he became bedridden. The patient died at the age of 27 because of pneumonia, although MLD patients usually die at the age of 40$50]$.

The analysis of this clinical case allowed making a diagnosis of an adult form of MLD. Biochemically and clinically, the diagnosis was confirmed by the exception of adrenoleukodystrophy, of which an excess of very long-chained fatty acids is typical, and some other forms of leukodystrophies. Some of the patient's neurological symptoms that were detected at the admission to the clinic (pyramidal, cerebellar, visual and pelvic disturbances) along with periventricular MRI of the brain white matter lesions demanded to exclude MS. However, the presence of the epileptic syndrome, rapidly increasing dementia, lack of intrathecal synthesis of immunoglobulins, the T2 MRI lesions symmetry and also the steadily progressing disease course without remissions made the diagnosis of MS doubtful and required the further examination of the patient.

In this patient, there were no symptoms of a hereditary disease like cerebral autosomal-dominant arteriopathy with subcortical infarctions and leukoencephalopathy, where repeated strokes, migraine-like headaches along with dementia or mental disorders could be observed.

The detection of an extremely low level of the crucial brain trophic factor BDNF also indicated the degenerative but not inflammatory character of the disease.

MLD (OMIM 250100) is attributed to the group of lysosomal storage disorders [21] and is a result of decreasing or complete loss of ASA lysosomal enzyme (EC 3.1.6.1) activity or in less frequent cases of its activator protein (sapozyne B) disturbance [11; 13]. A decrease of enzyme activity leads to a storage of nonhydralized granular metachromaticaly coloured substrate in brain cells and peripheral nerves. The lysosomes accumulation process results in the death and progressive degeneration of neurons and glyocytes [9].

In the only study, demyelination manifestations were considered to be specific for MLD [6] and that is why we consider that the specific pictures of demyelination detected in our study (Fig. 4 A, B) may have some typical signs just for MLD. The presence of macrophages and cells, looking like "fat" cells, in the biopsy material probably indicates an increase of the cells detoxication processes with lysosomal activation [12]. 
Detailed light microscopy morphological analysis of the biopsy material allowed to detect a practically complete lack of inflammation: there were not revealed any eosinophilic inclusions but there were some degenerative changes in the brain white matter that were characteristic for leukodystrophy. Electron microscopic examination also showed the evidence of neurodegenerative disease that appeared as axon damage (axonopathy) both in myelin and in non-myelin fibers, and as myelinopathy, that corresponded with the experimental and clinical data received earlier $[19 ; 8 ; 17 ; 5 ; 20]$. The patient's disturbances of the synapse structure also suggest some neurodegenerative changes.

In the patient's MRI scans symmetrical periventricular demyelination zones without contrast enhancement or any other inflammation signs were revealed and that considerably differed from MS picture. The similar picture of symmetrical hyperintensive signals on T2 weighted imaging was previously found in many variants of leukodystrophy [18].

The patient's biochemical and molecular-genetic tests revealed a considerable decrease of the activity of ASA, which is a significant marker of MLD [8]. It confirmed the diagnosis.

Consent: Informed consent was obtained from the parents of the patient to reproduce his case in this report.

Conflict of interests: The authors declare that they have no conflict of interests.

Disclaimer: The manuscript has not been published previously and is not under consideration for publication in any other journal.

\section{References}

1. Argyrakis, H. Pilz, H. H. Goebel, and D. Müller, "Ultrastructural findings of peripheral nerve in a preclinical case of adult metachromatic leukodystrophy," J Neuropathol. Exp. Neurol., vol. 36, no. 4, pp. 693-711, 1977.

2. M. Baraitser, "Metabolic disorders/degenerative diseases of childhood," in The Genetics of Neurological Disorders, 3rd ed, Oxford, Oxford Med. Publication, pp. 283-233, 1997.

3. A. Bardosi, R.L. Friede, S. Ropte, and H. H. Goebel, "A morphometric study on sural nerves in metachromatic leukodystrophy," Brain, vol.110, pp. 683-694, 1987.

4. Y. Q. Chen, M. A. Rafi, G. de Gala et al., "Cloning and expression of cDNA encoding human galactocerebrosidase, the enzyme deficient in globoid cell leukodystrophy," Hum. Mol. Genet., vol. 2, pp.1841$1845,1993$.

5. M. Eckhardt, K. Khalaj Hedayati, and J. Pitsch, "Sulfatide in neurons causes hyperexitability and axonal degeneration in a mouse model of metachromatic leukodystrophy," J. Neuroscience, vol. 27, no. 34, pp.9009-9021, 2007.

6. P. L. Faust, E. M. Kaye, and J. M. Powers, "Myelin lesions associated with lysosomal and peroxisomal disorders," Expert Rev.Neurother., vol. 10, no. 9, pp. 1449-1466, 2010.

7. Yu. M. Ghabotinsky and V. F. Sheffer, "Pathogenese and pathomorphology of human leukodystrophies," Arch. pathology, vol. 43, no. 11, pp. 86-92, 1981.

8. V. Gieselmann, A. Polten, J. Kreysing et al., "Arylsulfatase A pseudodeficiency: loss of a polyadenylylation signal and N-glycosylation site," Proc. Natl. Acad. Sci., vol. 86, pp. 9436-9440, 1989.

9. H. H. Goebel, A. Argyrakis, K. Shimokawa et al., "Adult metachromatic leukodystrophy. IV. Ultrastructural studies on the central and peripheral nervous system," Eur. Neurol., vol. 19, no. 5, pp. 294-307, 1980.

10. M. Hirano, and S. G. Pavlakis, "Mitochondrial myopathy, encephalopathy, lactic acidosis, and strokelike episodes (MELAS): current concepts," J. Child Neurol., vol. 9, pp. 4-13, 1994.

11. H. Holtschmidt, K. Sandhoff, H. Y. Kwon et al., "Sulfatide activator protein. Alternative splicing that generates three mRNAs and newly found mutation responsible for a clinical disease," J. Biol. Chem., vol. 266, pp.75567560, 1991.

12. E. Joosten, M. Hoes, A. Gabreëls-Festen et al., "Electron microscopic investigation of inclusion material in a case of adult metachromatic leukodystrophy; observations on kidney biopsy, peripheral nerve and cerebral white matter," Acta Neuropathol., vol. 33, no. 2, pp. 165-171, 1975.

13. E. H. Kolodny, and A. L. Fluharty, "Metachromatic leukodystrophy and multiple sulfatase deficiency: sulfatide lipidosis," in: Scriver C.R., Beaudet A.L., Sly W.S. et al., eds. The metabolic and molecular bases of inherited disease, 7th ed., vol. 2., N.Y., McGraw-Hill, pp. 2693-2739, 1995. 
14. K. D. Krasnopolskaya, "Genetic deseases of metabolism", Referencial text-book for physicians, M., ROO «Centre of social kinder adaptation and rehabilitation », 2005, 364p.

15. G. A. Merkulov, "Course of pathohistological techniques", Leningrad: Medgiz, 1969, 424p.

16. A.A. Mironov, Ya. Yu. Comissarchik, and V. A. Mironov, "Electron microscopy in medicine and biology," S.-Pb, Science, 1994, 400p.

17. M. Molander-Melin, Z. Pernber, S. Franken et al., "Accumulation of sulfatide in neuronal and glial cells of arylsulfatase a deficient mice," J. Neurocytol., vol. 33, pp. 417-427, 2004.

18. Z. Patay, "Diffusion-weighted MR imaging in leucodystrophies," Eur.Radiol., vol.15, pp. 2284-2303, 2005.

19. L.Peng, and K. Suzuki, "Ultrastructural study of neurons in metachromatic leukodystrophy," Clin. Neuropathol., vol. 6, no. 5, pp. 224-230, 1987.

20. H. Ramakrishnan, K.K. Hedayati, R. Lüllmann-Rauch et al., "Increasing sulfatide synthesis in myelin-forming cells of arylsulfatase A-deficient mice causes demyelination and neurological symptoms reminiscent of human metachromatic leukodystrophy," J. Neurosci., vol. 27, no. 35, pp. 9482-9490, 2007.

21. J. Rapola, "lysosomal storage diseases in adults," Pathol. Res. Pract., vol. 190, no. 8, pp. 759-766, 1994.

22. H. J. Sommerlade, A. Hille-Rehfeld, K. von Figura et al., "Four monoclonal antibodies inhibit the recognition of arylsulphatase A by the lysosomal enzyme phosphotransferase," Biochem. J., vol. 297, pp. 123-130, 1994. 